\title{
„ES WAR EIN SCHÖNER AUGUSTTAG DES JAHRES 1913.“ LUFTTEMPERATUR UND GESELLSCHAFTLICHE UMBRUCHSSITUATION IN ROBERT MUSILS DER MANN OHNE EIGENSCHAFTEN
}

\begin{abstract}
It was a fine day in August 1913." Air Temperature and Societal Upheaval in Robert Musil's "The Man Without Qualities"

In Robert Musil's fragmentary novel, The Man Without Qualities, history and poetics connect in a very potent manner. A salient example of this is the famous sentence, "It was a fine day in August 1913", where societal upheaval is portrayed. Meteorological information contrasts and corresponds with scientific facts and documented historical events. Above all, it is Musil's ironic style which brings the text's literary power of commentary to the fore.
\end{abstract}

KEYWORDS: Robert Musil, First World War, Temperature, Weather, Knowledge/Science, Meteorology

Robert Musils Romanprojekt Der Mann ohne Eigenschaften kann als gedankliches Experiment gelten, in dem mittels eines historischen Rückgriffs Gegenwart erklärbar wird und sich zugleich ein seismographisches Gespür für kommende Gewaltereignisse abzeichnet. Der Roman, veröffentlicht mit seinem ersten Band 1930, steht auf der Schwelle zu der Machtergreifung des Nationalsozialismus und des drohenden Zweiten Weltkrieges. Die Welten, die dieses umfangreiche narrative Konvolut aufwirft, beschränken sich dabei zwar auf einen prägnanten historischen Rückblick, nehmen aber im Erzählen die ultimative Katastrophe vorweg. Die Handlung beginnt im August 1913 und wird im Verlauf des narrativen Geschehens zeitlich nur langsam voranschreiten. So wird nach weit über Tausenden von Seiten, die Musil in den nächsten Jahren bis zu seinem Tod am 15. April 1942 niederschreiben sollte, erst das Ende des Jahres 1913 erreicht sein. ${ }^{1}$ Der sogenannte Große Krieg von

\footnotetext{
${ }^{1}$ Vgl. Karl Corino: Robert Musil. Eine Biographie. Reinbek b. Hamburg 2003, S. 1438. Die von Adolf Frisé 1978 neu durchgesehene und verbesserte Ausgabe umfasst allein über 2000 Seiten. Die digitale Klagenfurter Ausgabe von 2009 weit mehr als 11.000 Seiten.
} 
1914 bis 1918 findet sich zwar im Text vorbereitet, liegt aber für Musils Schreibarbeit noch in weiter Ferne.

Herfried Münkler weist in seiner Studie zum Ersten Weltkrieg auf den amerikanischen Diplomaten und Historiker George F. Kennan hin, der diesen Krieg als die ,Urkatastrophe des 20. Jahrhunderts' bezeichnet hat. ${ }^{2}$ Der Roman Der Mann ohne Eigenschaften beschreibt das politisch überholte Österreich-Ungarn in seiner späten Phase und zeigt, welche Auswirkungen diese gesellschaftliche Lähmung für ein Vorkriegseuropa hat, das schon bald seinen Zusammenbruch erleben wird. Zugleich ist Musils Fokussierung auf die k.u.k. Monarchie ein Abgesang auf eine multikulturelle und vielsprachige Gesellschaft, deren Modernität in dieser pluralen Form sein Ende finden wird. Wie nun setzt Musil diese ,Zeitenschwelle ${ }^{3}$ in Szene, wie inszeniert er eine sinnliche Qualität, die den Leser in seinen Bann ziehen wird? Und wie verhalten Historizität und Narrativität sich zueinander?

\begin{abstract}
Über dem Atlantik befand sich ein barometrisches Minimum; es wanderte ostwärts, einem über Rußland lagernden Maximum zu, und verriet noch nicht die Neigung, diesem nördlich auszuweichen. Die Isothermen und Isotheren taten ihre Schuldigkeit. Die Lufttemperatur stand in einem ordnungsgemäßen Verhältnis zur mittleren Jahrestemperatur, zur Temperatur des kältesten wie des wärmsten Monats und zur aperiodischen monatlichen Temperaturschwankung. Der Auf- und Untergang der Sonne, des Mondes, der Lichtwechsel des Mondes, der Venus, des Saturnringes und viele andere bedeutsame Erscheinungen entsprachen ihrer Voraussage in den astronomischen Jahrbüchern. Der Wasserdampf in der Luft hatte seine höchste Spannkraft, und die Feuchtigkeit der Luft war gering. Mit einem Wort, das das Tatsächliche recht gut bezeichnet, wenn es auch etwas altmodisch ist: Es war ein schöner Augusttag des Jahres $1913 .^{4}$
\end{abstract}

Mit diesen Worten beginnt Robert Musils fundamentales Romanprojekt. Neben Prousts Auf der Suche nach der verlorenen Zeit (1913) und James Joyces Ulysses (1922) ist Der Mann ohne Eigenschaften das dritte große Werk, das die Moderne mit ihrer ungeheuren Sprengkraft des Jahres 1913 zum Ausdruck bringt. Robert Musils unvollendetes Textkonvolut beginnt an einem Augusttag des Jahres 1913; einer Zeit, in der die Krise eine Art Lebensgefühl darstellt. Zugleich ist diese Wirklichkeit gezeichnet von sozialen Widersprüchen und politischen Lähmungen, für die man im Nachhinein den Eindruck gewinnen könnte, dass nur Weniges zu passieren hätte, um eine größere Katastrophe hervorzubringen. Doch zielt der poetische Ton, den Musil hier wählt, weniger auf die Schwere einer historischen Faktizität, sondern beschreibt eher in einer Mischung aus ästhetischer Unklarheit und naturwissenschaftlicher Genauigkeit und Sorgfalt einen seltsamen Schwebezustand. Ja, man könnte vielleicht sogar behaupten, dass hier eine Zeitdimension angestrebt wird, die

\footnotetext{
${ }^{2}$ Vgl. Herfried Münkler: Der Große Krieg. Die Welt 1914 bis 1918. Berlin 2013, S. 9.

${ }^{3}$ Ein Begriff, der auf Dan Diner zurückgeht. Vgl. Dan Diner: Zeitenschwelle. Gegenwartsfragen an die Geschichte. München 2010.

${ }^{4}$ Robert Musil: Der Mann ohne Eigenschaften. 2 Bde. Reinbek b. Hamburg 1987, S. 9.
} 
mittels einer Leichtigkeit als vorherrschendes Stilmittel auf nicht verwirklichte Möglichkeiten anspielt. Profanes mischt sich mit kosmogonischen, mythischen Bildwelten und entzieht dieser Eingangsszene jegliche Bedeutungsschwere. An einem solchen Tag - der hier eben als wunderschöner Sommertag beschrieben wird - beschließt der Intellektuelle Ulrich, sich für ein Jahr frei zu nehmen, nachdem ein weiterer seiner Versuche, eine berufliche Karriere einzuschlagen, gescheitert scheint.

Wichtig ist in diesem Zusammenhang, die in der Eröffnungspassage des Romans anzitierten wissenshistorischen Überlegungen näher zu betrachten und genauer zu analysieren, worauf Musil hier rekurriert. Denn es ist evident, dass für das Werk Der Mann ohne Eigenschaften neben dem Aspekt einer allgemeinen Faktizität und Funktionstüchtigkeit des Gesagten ebenfalls das Erzeugen einer leichten Schreibweise im Vordergrund steht. Die Besonderheit besteht darin, eine ,Literarizität von Wissen' zu erzeugen, wie Florian Kappeler in seiner Studie zu Musil so treffend bemerkt. ${ }^{5}$ Kappeler weist zu Recht darauf hin, dass in der Eingangsszene von Musils Roman eine „meteorologische Terminologie mit einer poetischen Wetterbeschreibung korrespondiert" ${ }^{\text {"6 }}$ und zwei Begriffssysteme quasi amalgamiert werden. Dabei unterscheidet er zwischen dem Wissenssubjekt und -objekt, wobei er darlegt, dass die Berichterstattung des Wissenssubjekts ohne Weiteres den üblichen Wetterberichten entspricht. Auffallend und interessanter ist dagegen die Objektseite. Denn das meteorologische Vokabular scheint vielmehr „eine Hochdruck-Wetterlage im Winter $^{\text {“ } 7}$ zu beschreiben, während das literarische Begriffssystem auf ,einen schönen Sommertag' zielt. Diese Ungereimtheit wird aber für den Leser nicht notwendig relevant sein, denn wozu sollte es das hier vorgestellte Wissen hinterfragen. Doch wird er vermutlich der literarisch-ästhetischen Spur folgen und den ,schönen Sommertag' ohne Weiteres genießen, aber auch kritisch reflektieren. ${ }^{8}$

Aber wie war das Wetter denn wirklich in diesen Augusttagen des Jahres 1913 in Wien? In der Neuen Freien Presse erscheint am 15. August ein ausführlicher Artikel mit der Überschrift „Ausdauerndes schlechtes Wetter“, das von dem Assistenten der Zentralanstalt für Meteorologie Dr. O. Freiherrn von Myrbach verfasst wurde. Es heißt hier wie folgt:

Wie zu befürchten war, hat das heurige Sommerwetter im wesentlichen den Charakter treulich beibehalten, den es von Anfang an trug. Seine Härten haben freilich etwas nachgelassen und sind einer

\footnotetext{
${ }^{5}$ Vgl. Florian Kappeler: Situiertes Geschlecht. Organisation, Psychiatrie und Anthropologie in Robert Musils Roman „Der Mann ohne Eigenschaften“. München 2012, S. 10.

${ }^{6}$ Ebd., S. 31.

${ }^{7}$ Ebd., S. 32.

${ }^{8}$ Vgl. allg. zur Bedeutung von Klima und Wetter: Ronald D. Gerste: Wie das Wetter Geschichte macht. Katastrophen und Klimawandel von der Antike bis heute. Stuttgart 2015. Gerste untersucht Wetterepisoden und Klimaschwankungen in ihren Auswirkungen auf Gesellschaft und Kultur. Dabei zeigt er auf, wie das Wetter zum Marker von geschichtlichen Ereignissen werden kann. Das Jahr 1914 und der erste Weltkrieg finden jedoch bei ihm keine Erwähnung.
} 
sehr langsam wirkenden, aber doch immer mehr fortschreitenden Besserung gewichen. Das will aber noch nicht viel sagen, denn der Beginn des Sommers war so außergewöhnlich schlecht, daß auch die spätere Zeit trotz der Besserung noch als schlecht bezeichnet werden muß.

Die sekundäre Hauptursache des vorwiegend regnerischen und vor allem jetzt kühlen Wetters ist die Erhaltungstendenz des Hochdruckgebietes im Nordwesten Europas und weiterhin die Zyklonen bildende Wirkung des Gebietes zwischen der Ostsee und dem Schwarzen Meer. Unter diesen Umständen hatte auch das Gebiet südlich der Alpen eine große Neigung, Tiefdruckgebiete zu erzeugen, die durch ihre Entwicklung immer eine ganz besondere Verschlechterung des Wetters brachten. Wie sehr der fast ständige westöstliche Luftdruckgradient mit seinen nördlichen Winden die Wärme Mitteleuropas beeinträchtigt, zeigt der Umstand, daß die mittlere Julitemperatur in Wien um 3'6 Grad Celsius unternormal blieb. Natürlich mußten auch die fast immer mit einer nördlichen Komponente auftretenden Winde beim Uebersetzen der Alpenkette gewaltige Wassermassen zurücklassen. So fielen in Ischl seit Beginn des Sommers 518 Millimeter Regen. ${ }^{9}$

Was heißt das? Darauf hat Florian Illies in seinem Buch 1913. Der Sommer des Jahrhunderts (2014) hingewiesen. Es bedeutet schlicht: Es gab keinen schönen Augusttag des Jahres 1913. Denn in Wien herrschte eine Durchschnittstemperatur von 16 Grad Celsius. Insofern kann der August überhaupt als der kälteste August des gesamten Jahrhunderts gelten. In diesem Zusammenhang weist Illies darauf hin, dass es allzu gut war, „dass das die Menschen 1913 noch nicht wussten““10. Außerdem kam neben der Kälte dann noch der Regen hinzu. So weiß das Wiener Fremden-Blatt am Freitag, dem 15. August 1913, in Bezug auf einen möglichen „Sommerbummel“" Folgendes zu berichten:

Eigentlich müßte man dazu sagen: im Regen. Denn der heurige Sommer übertrifft in dieser Hinsicht alles bisher Dagewesene und die bekanntesten ältesten Leute können sich nicht erinnern u.s.w. Regen des Morgens, Regen zu Mittag und Regen des Abends und damit etwas Abwechslung kommt, in den Zwischenpausen einige Kneippsche Güsse erster und zweiter Ordnung, bis die dunklen Abendwolken die trostlose Symphonie in Grau langsam in sich aufnehmen. Und noch im Bett hört man die schweren dicken Tropfen klitsch-klatsch an die Fenster schlagen und denkt mit Schaudern daran, daß der morgige Tag wieder vierundzwanzig Stunden hat, die doch auf irgendeine Art totgeschlagen werden müssen. Aber wie? Gut, man kann zwölf Stunden davon verschlafen und einigermaßen einholen, was man in den Monaten vorher versäumt hat -, bleiben aber noch immer zwölf nach Adam Riese, und zwölf Stunden [...] sind eine erschreckend lange Zeit [...]. ${ }^{11}$

Dass es damals am Augusttag des Jahres 1913 geregnet hat, davon gibt der Comic Der Mann ohne Eigenschaften (2013) des Zeichners Nicolas Mahler ebenfalls Auskunft. Denn hier sehen wir den Mann ohne Eigenschaften immer mit einem Regen-

${ }^{9}$ Dr. O. Freiherr von Myrbach: Andauerndes schlechtes Wetter. In: Neue Freie Presse, Nr. 17593, 15.08.1913, Morgenblatt, S. 11.

${ }^{10}$ Florian Illies: 1913. Der Sommer des Jahrhunderts. Frankfurt a. M. 2014, S. 199.

${ }^{11}$ R. K.: Sommerbummel. In: Fremden-Blatt. 67. Jg., Nr. 233, Wien, Freitag, 15. August 1913, Morgen-Blatt, S. 6. 
schirm herumlaufen ${ }^{12}$; ja, es scheint, dieser Schirm gehört einfach zu diesem ,Jahrhundertsommer', der eben keiner war.

Wozu also dieser ganze Aufwand mit den zahlreichen meteorologischen Angaben und der Inszenierung einer scheinbar schön-warmen Temperatur im August? Handelt es sich um ein seismographisches Gespür für den Großen Krieg? Um ein ironisches Stilmittel? Ist es - wie Hans Ulrich Gumbrecht bemerkt - ausschließlich die Aufgabe dieser einführenden Textpassage, dass wir plötzlich eine vergessene Welt nacherleben, sie geradezu durch die Temperaturangabe ,von innen" wahrzunehmen vermögen ${ }^{13}$ Soll diese behagliche Temperatur uns einstimmen auf das, was dann folgt? Auffallend ist, dass Musil den ,schönen Augusttag“ - zeitlich gesehen vor die ,Urkatastrophe des 20. Jahrhunderts' setzt. Die warme, angenehme Temperatur, die Flüchtigkeit eines vorübergehenden schönen Tages, die Leichtigkeit des Seins steht im Kontrast zum Großen Krieg, der die Welt in ihren Grundfesten erschüttern sollte. Denn der Erste Weltkrieg - so zeigt die Studie von Münkler - sollte nicht ausschließlich vom Zweiten her betrachtet werden, sondern muss ,als ein für sich alleine stehendes, komplexes Ereignis ${ }^{\text {“14 }}$ behandelt werden, das bis heute zahlreiche politische Konflikte und Probleme aufwirft. Hierzu zählt Münkler die Ordnung in Mittel- und Osteuropa in all ihrer Instabilität, selbst noch im pazifischen Raum entstehen Krisenpotenziale, die sich im Zweiten Weltkrieg manifestieren, aber auch noch weit darüber hinausreichen sollten. Das größte Problem aber, das der Erste Weltkrieg hervorruft, ist der postimperiale Raum des Nahen und Mittleren Ostens, der in der geopolitischen Terminologie der USA heute ,Greater Middle East ${ }^{\star}$ heißt. ${ }^{15}$ Zugleich war der Erste Weltkrieg - und darauf hat Münkler ebenfalls hingewiesen - der erste Krieg, in dem die Intellektuellen eine einflussreiche Rolle spielen sollten. ${ }^{16}$ „Die Deutungseliten“ - wie Münkler sie nennt - „haben sich nachhaltig in das Geschäft der Entscheidungseliten eingemischt und dabei haben sie mehr zur Eskalation als zur Moderation des Kriegsgeschehens beigetragen ““17.

Könnte der schöne Sommertag also als Kontrastfolie zum schrecklichen Krieg fungieren, ist damit aber noch längst nicht alles zu dieser Textpassage gesagt. Übrigens hat Helmut Lethen in seiner Monographie Verhaltenslehren der Kälte für die

\footnotetext{
${ }^{12}$ Vgl. Musil/Mahler: Der Mann ohne Eigenschaften. Gezeichnet von Nicolas Mahler. Berlin 2014. Der Regenschirm ist hier fast auf jeder Seite zu sehen.

${ }^{13}$ Vgl. Hans Ulrich Gumbrecht: Robert Musil erlösen! In: Frankfurter Allgemeine Zeitung, 21. Januar 2012. Vgl. allg. dazu auch: Hans Ulrich Gumbrecht: Stimmungen lesen. Über eine verdeckte Wirklichkeit der Literatur. München 2011. In seiner Monographie Stimmungen lesen beschreibt er u. a. eine Form des Lesens, die auf den Körper zielt und sich dabei unmittelbar mit dem Wetter verbinden kann. Vgl. S. 11.

${ }^{14}$ Münkler: Der Große Krieg, S. 11.

${ }^{15} \mathrm{Vgl}$. ebd.

${ }^{16}$ Ebd., S. 17.

${ }^{17}$ Ebd., S. 18.
} 
Beschreibung des Zusammenbruchs der k.u.k. Monarchie als Ausgangsbild für den Ersten Weltkrieg eine Kältemetaphorik benutzt, indem er auf Manès Sperbers Erinnerungen und Reflexionen zurückgreift. ${ }^{18}$ Ich zitiere Sperber:

Es war im Jahr 1918, am Vormittag des ersten oder zweiten November, auf einem Bahnsteig des Wiener Nordbahnhofs. Seit einer Woche trieb ich mich dort herum, verbrachte meine Zeit mit bald hoffnungsvollem, bald verzweifeltem Warten. Mein Vater hatte seine Heimkehr angekündigt, doch die Unordnung im Zugsverkehr war chaotisch geworden, Fahrpläne galten nicht mehr. Der Vater mochte in der nächsten Minute oder erst nach Tagen eintreffen.

Es war gegen elf Uhr vormittags, ein kalter Wind blies über die Bahnsteige, doch verminderte sich die Zahl der Leute nicht, die auf Züge warteten, die nicht ankamen oder nicht abfuhren, auf Urlauber, die vielleicht nicht mehr lebten, auf Verwandte, die auf einer Umsteigestation steckengeblieben waren. Die meisten aber waren Soldaten auf dem Weg zu ihren Einheiten; sie hatten es nicht eilig, lungerten herum und fanden sich mit der Verspätung gerne $a b .{ }^{19}$

Wärme contra Kälte - die Inszenierungen von Temperaturen also, um einschneidende politische Katastrophen und Geschehen sowie existenzielle Krisenereignisse zum Ausdruck zu bringen? Geht das wirklich so auf?

Roland Barthes hat darauf aufmerksam gemacht, dass das Wetter und die Jahreszeiten sich unmittelbar auf das Schreiben eines Romans auswirken. Am Beispiel des japanischen Haikus zeigt er, dass diese literarische Form angibt und damit deutlich markiert, wo man sich als Leser oder Zuhörer im Jahresverlauf zu verorten hat.

Im Haiku gibt es immer etwas, das Ihnen sagt, wo Sie sich im Jahresverlauf befinden, unter welchem Himmel, in welcher Kälte und in welchem Licht. [...] Immer spüren Sie die Jahreszeit: als Fluidum und Zeichen zugleich. ${ }^{20}$

Und genau auf einen solchen Zusammenhang spielt Musil mit seinem Bericht zur atmosphärischen Lage über Mitteleuropa an. Wie wir gesehen haben, ist die $\mathrm{Zu}$ sammenstellung von Versatzstücken aus Wissensdiskursen der Geographie, Meteorologie, Astronomie, Kultur und Ästhetik vermischt mit Überlegungen des Alltags, eine Besonderheit, die jegliche Aussage letztlich unterläuft. So verweist Inka Mülder-Bach in ihrer Studie zu Musil (2013) darauf, dass etwa dem „barometrischen Minimum“, das sich über dem Atlantik „befand“, eine zukünftige „Neigung“ angedichtet, den „Isothermen und Isotheren“ hingegen eine „Schuldigkeit“ unterstellt wird. ${ }^{21}$ Es ist also die Ironie, die hier in Szene gesetzt wird und einer Amalgamie-

${ }^{18}$ Vgl. Helmut Lethen: Verhaltenslehren der Kälte. Lebensversuche zwischen den Kriegen. Frankfurt a. M. 1994, S. 16.

${ }^{19}$ Manès Sperber: Leben in dieser Zeit. Sieben Fragen zur Gewalt. Wien 1972, S. 9.

${ }^{20}$ Roland Barthes: Die Vorbereitung des Romans. Vorlesung am Collège de France. 1978-1979 und 1979-1980. Deutsch von Horst Brühmann. Hrsg. von Éric Marty. Frankfurt a. M. 2008, S. 78 [Kursive im Original].

${ }^{21}$ Vgl. Inka Mülder-Bach: Robert Musil. „Der Mann ohne Eigenschaften“. Ein Versuch über den Roman. München 2013, S. 24. 
rung von Wissenschaften und Literatur Vorschub leistet. Es ist vor allem die Beschreibung einer gemischten Lage, die etwas Unbestimmtes hervorbringt. Ein Erzähleinsatz also, der in seinem ironischen Ton nicht nur die Wissensdiskurse karikiert und persifliert, sondern das Literarische selbst.

Dass Musil sich in dieser Textpassage auf Ludwig Klages' Vom kosmogonischen Eros (1922) bezieht, darauf hat Inka Mülder-Bach ebenfalls hingewiesen. Denn Klages bringt als Gegenentwurf zum ,Trieb“ den Begriff der ,Neigung' ins Spiel, der einen ,Hang', eine ,Hingezogenheit‘ bezeichnet. ${ }^{22}$ Diese Gefühlsdimensionen einer Eros-Theorie mischt Musil nun mit dem Wetterbericht. ,Neigung' kommt jetzt einem energetischen System zu, das sich, wie der Luftdruck, in atmosphärischen Formen aufbaut, das steigt oder sinkt und das beim tiefsten Stand eines barometrischen Minimums sich zum Sturm steigern kann. Die ironische Wendung ist bei Musil also deutlich. Alles wird hier in einer ,trichterförmigen' Zuspitzung verortet wie Norbert Wolf es in seiner Monographie Kakanien als Gesellschaftskonstruktion (2011) sieht. Aus einer freischwebenden Erzählerwarte wird alles ,,von der meteorologischen Globalperspektive der Stratosphäre in die Straßen der Großstadt Wiens“23 in Szene gesetzt. Eine narrative Kamerafahrt also von oben nach unten, die an einem schönen „Augusttag des Jahres 1913“ endet. Alexander Honold spricht in diesem Zusammenhang auch von dem point of view, dem „Auge“ des Erzählers in einer „Abstraktionshöhe“. ${ }^{24}$ Dass dieser Sommertag in Wirklichkeit keiner war, macht diesen ironischen Textanfang umso bemerkenswerter, trägt doch das erste Kapitel des Mannes ohne Eigenschaften den sprechenden Titel: Woraus bemerkenswerter Weise nichts hervorgeht. ${ }^{25}$ Und was heißt das für das Begehren? Eben dass es auf der Suche ist.

\section{Literatur}

Barthes, Roland: Die Vorbereitung des Romans. Vorlesung am Collège de France. 1978-1979 und 1979-1980. Deutsch von Horst Brühmann. Herausgegeben von Éric Marty. Frankfurt a. M. 2008.

Corino, Karl: Robert Musil. Eine Biographie. Reinbek b. Hamburg 2003.

Diner, Dan: Zeitenschwelle. Gegenwartsfragen an die Geschichte. München 2010.

Gerste, Ronald D.: Wie das Wetter Geschichte macht. Katastrophen und Klimawandel von der Antike bis heute. Stuttgart 2015.

Gumbrecht, Hans Ulrich: Stimmungen lesen. Über eine verdeckte Wirklichkeit der Literatur. München 2011.

\footnotetext{
${ }^{22}$ Vgl. ebd.

${ }^{23}$ Norbert Christian Wolf: Kakanien als Gesellschaftskonstruktion. Robert Musils Sozioanalyse des 20. Jahrhunderts. Wien/Köln/Weimar 2011, S. 264.

${ }^{24}$ Alexander Honold: Einsatz der Dichtung. Literatur im Zeichen des Ersten Weltkrieges. Berlin 2015, S. 697.

${ }^{25}$ Musil: Der Mann ohne Eigenschaften, S. 9.
} 
Gumbrecht, Hans Ulrich: Robert Musil erlösen! In: Frankfurter Allgemeine Zeitung, 21. Januar 2012. Honold, Alexander: Einsatz der Dichtung. Literatur im Zeichen des Ersten Weltkriegs. Berlin 2015. Illies, Florian: 1913. Der Sommer des Jahrhunderts. Frankfurt a. M. 2014.

Kappeler, Florian: Situiertes Geschlecht. Organisation, Psychiatrie und Anthropologie in Robert Musils Roman „Der Mann ohne Eigenschaften“. München 2012.

Lethen, Helmut: Verhaltenslehren der Kälte. Lebensversuche zwischen den Kriegen. Frankfurt a. M. 1994.

Musil, Robert: Der Mann ohne Eigenschaften. 2 Bde. Reinbek b. Hamburg 1987.

Musil, Robert: Klagenfurter Ausgabe. Kommentierte digitale Edition sämtlicher Werke, Briefe und nachgelassener Schriften. Mit Transkriptionen und Faksimiles aller Handschriften. Hrsg. von Walter Fanta, Klaus Amann und Karl Corino. Klagenfurt 2009.

Musil/Mahler: Der Mann ohne Eigenschaften. Gezeichnet von Nicolas Mahler. Berlin 2014.

Mülder-Bach, Inka: Robert Musil. „Der Mann ohne Eigenschaften“. Ein Versuch über den Roman. München 2013.

Münkler, Herfried: Der Große Krieg. Die Welt 1914 bis 1918. Berlin 2013.

Myrbach, Dr. O. Freiherr von: Andauerndes schlechtes Wetter. In: Neue Freie Presse. Nr. 17593, 15.08.1913, Morgenblatt, S. 11-12.

R. K.: Sommerbummel. In: Fremden-Blatt. 67. Jg., Nr. 223, Wien, Freitag, 15. August.1913, MorgenBlatt, S. 6-7.

Rückenbacher, Sergej: Der Fehler, kein Buch zu sein. Die Klagenfurter Robert Musil-Ausgabe und die ästhetische Erfahrung des Buches. In: Massimo Salgero (Hrsg.): Robert Musil in der Klagenfurter Ausgabe. Bedingungen und Möglichkeiten einer digitalen Edition. München 2014, S. 173-195.

Sperber, Manès: Leben in dieser Zeit. Sieben Fragen zur Gewalt. Wien 1972.

Wolf, Norbert Christian: Kakanien als Gesellschaftskonstruktion. Robert Musils Sozioanalyse des 20. Jahrhunderts. Wien/Köln/Weimar 2011. 